\title{
LA EDUCACIÓN SUPERIOR PRIVADA EN BRASIL ${ }^{1}$
}

\author{
Claudio Rama \\ (Dr. ED; Dr. DER $)^{2}$ \\ Universidad de la Empresa (UDE), Uruguay
}

\section{RESUMEN}

La dinámica de la educación superior en Brasil se constituye como un escenario muy propio e el marco de la región de América Latina. Su génesis, su tamaño y su propia estructuración son una especificidad. Sin embargo, en el actual contexto, ella esta mostrando nuevas configuraciones dada por el nivel de concentración del sector y su grado de internacionalización, que se constituyen como nuevas modalidades de funcionamiento de las dinámicas de la educación superior. El presente ensayo, parte de un estudio más amplio, inserta y analiza las nuevas características de este sector

Palabras claves: Educación superior privada; América Latina; concentración; mercados universitarios; tendencias universitarias

\section{PRIVATE HIGHER EDUCATION IN BRAZIL}

\section{ABSTRACT}

The dynamics of higher education in Brazil is a very typical scenario and the context of Latin America. Its genesis, its size and structure are very specific. However, in the present context, it is showing new configurations given by the level of industry concentration and the degree of internationalization, which are constituted as new modes of operation of the dynamics of higher education. This essay, part of a larger study, inserts and analyzes the new features in this area

Keywords: Private Higher Education; Latin America; concentration; university markets; trends university

\section{Indice}

1. La estructuración histórica de la educación superior privada. 2. Dimensión académica como instrumento de regulación. 3. Ejes políticos de la educación superior privada. 4. Dimensión social de las universidades privadas. 5. Dimensión económica de la educación superior. 6. Tendencias y conclusiones

\section{La estructuración histórica de la educación superior privada}

El país tiene un modelo de organización institucional del sector terciario de tipo dual articulado sobre la base de una educación pública de acceso gratuito, basada tradicionalmente en altos estándares de calidad derivados del carácter selectivo, de los salarios docentes, del peso de la postgraduación y de una orientación hacia la investigación. Algunos fijan desde la reforma de Getulio Vargas en 1938 un modelo del sector público orientado bajo el patrón de las Universidades americanas focalizadas en respuestas especializadas y orientadas al mercado bajo un modelo de investigación de tipo alemán. ${ }^{3}$ Tanto en el acceso a los estudios de grado como a los de postgrado existen altos niveles de selección académicos, reafirmados por la gratuidad y por los altos estándares de calidad a su vez asociados a la estructura de las remuneraciones laborales. El acceso a la carrera docente en el sector federal y algunas universidades estaduales es también 
altamente competitivo y selectivo. El sector público tuvo históricamente una orientación hacia la investigación, articulado inclusive en una organización universitaria basada en Departamentos desde la reforma universitaria de fines de los sesenta, que entre otros componentes sustituyó las cátedras por los departamentos. ${ }^{4}$

Las universidades del sector público nacieron recién a comienzos del siglo XX como resultado de la unificación de las Facultades aisladas que habían sido creadas en el siglo XIX, como derivación de decisiones políticas de centralización de esas unidades académicas en el marco de modelo de elites y gratuito como en toda la región, pero mantuvo posteriormente su carácter de elite. Recién en el contexto de la década del 2000 en el marco del gobierno de izquierda se ha pretendido encarar una política de expansión del sector público y de relativa des-elitización diferenciada, y al tiempo de restricción y control sobre el sector privado, aunque en general aún sin resultados (2008). Complementariamente, la educación superior privada en Brasil tiene su tradición desde inicios del siglo XX. Ya entre 1910 y 1918 se implantaron 56 nuevas escuelas privadas en el marco del mismo modelo de facultades aisladas que prevaleció en el sector público. Fue también posteriormente que se comenzaron a crear Universidades propiamente tales como en 1941 la Universidad Católica de Río de Janeiro. El sector privado nació en forma independiente, asociado a instituciones filantrópicas y religiosas, y se orientó a una formación de profesionales de elites, en el marco de un sistema público de elites también. Desde sus inicios ambos sectores se desarrollaron conjuntamente, y coincidieron en sus génesis, en parte por el retraso en la creación de universidades públicas y la autorización a la creación de Universidades privadas. Ambos procesos se dieron recién significativamente en el siglo XX.

Esta dinámica integrada se verificaba para 1933 cuando la cobertura privada alcanzaba al $44 \%$ de los estudiantes terciarios y el $60 \%$ de las instituciones. ${ }^{5}$ El crecimiento del sector privado en Brasil tiene componentes estructurales y ha sido derivado de una larga evolución. El peso del sector privado pasó de 42,3\% en 1955, a 44,3\% en $1960 ; 43,8 \%$ en $1965,50,5 \%$ en $1970,61,8 \%$ en 1975 y $63,3 \%$ en 1980 . En este sentido si bien la construcción de un sistema de enseñanza público de educación ha sido una "conquista de este siglo", 6 y no fue una característica estructural o de la génesis del sistema universitario, sin embargo, el tendió a tener un menor peso relativo a medida que la cobertura aumentaba. Así, a pesar de tener relativamente orígenes conjuntos históricamente, la expansión de ambas modalidades fue diferenciada. En tanto el sector público se oriento hacia la calidad y hacia la formación de las elites con una muy temprana postgraduación en relación al resto de América Latina sobre la base de políticas públicas de incentivo y regulación articuladas crecientemente a través de CAPES que se constituyó en uno de los ejes distintivos del sistema universitario de Brasil. ${ }^{7}$ A diferencia el sector privado se oriento hacia la masificación de la cobertura. El sector público mantuvo su carácter de elite, en tanto que el sector privado, al tener que financiarse por el mercado en su totalidad, promovió la expansión del acceso asociado al propio aumento de las demandas y al carácter altamente selectivo del sector público.

Ello estructuró una dinámica que impulsó una alta participación del sector privado en la cobertura terciaria, con una fuerte tendencia hacia una absorción de la demanda para sector de menores niveles de capital cultural y de menores ingresos, lo cual a su vez, más allá de una alta diferenciación de la educación superior privada en el cual en la cúspide se colocaban unas pocas universidades de calidad, aunque inferiores a algunas del sector público, en general facilitó una alta estratificación de instituciones altamente diferenciadas que en muchos casos, para cubrir las demandas de acceso de nuevos sectores sociales, no se alcanzaban a cubrir los estándares de calidad que caracterizaban al sector público. Este 
tuvo históricamente una dinámica de crecimiento basada en la calidad de tipo selectivo, de acceso totalmente gratuito y que promovió un continuo proceso de incremento salarial de los docentes a partir de aumentos de la formación asociado a un incremento de los requisitos de acceso tanto para docentes como para estudiantes, en este caso a través de un examen que se llama vestibular que deben realizar las Universidades. La educación pública ha derivado en una estructura de costos muy superior a la del sector privado. ${ }^{8}$

El modelo selectivo de acceso a las instituciones públicas superiores es una característica desde la primera ley de educación superior en el país a comienzos del siglo XX. Muchos de estos exámenes son realizados por fundaciones que agrupan a diferentes universidades. ${ }^{9}$ En el sector privado no existen, salvo excepciones correspondientes al sector de acceso de elites, estos exámenes selectivos. Tal dinámica promovió un permanente aumento de la cobertura asociado a los ingresos de las familias que permitió colocar a sus hijos en la educación media privada y a su stock de capital cultural. Tal expansión fue siempre menor que el nivel de incremento del sector privado y fue conformando una dinámica creciente del sector privado.

El rol de CAPES creado en la década del 50 se constituyó en el instrumento fundamental de esta orientación de elites de calidad, siguiendo en general el modelo de universidades americanas de centros de investigación con financiamientos públicos competitivos externos adicionales. Esta dinámica, facilitó durante mucho tiempo, y aún lo continúa haciendo, la conformación de modelos de investigación y al tiempo una alta resistencia a la masificación de la educación superior pública en Brasil por parte de las propias estructuras docentes que han visualizado claramente la relación entre salarios docentes elevados, calidad y selectividad.

El incremento de la educación privada ha tenido connotaciones ideológicas, en tanto la educación pública, como en toda la región, se posiciono y se visualizó como el foco de resistencia social a las dictaduras y el espacio político de las luchas y las banderas de la democratización. Su carácter de elite fue un mecanismo por el cual también se reafirmó en Brasil, la diferenciación entre los cuadros políticos intelectuales y los sectores populares. A diferencia la educación privada siempre tuvo un perfil gubernista, y dado el carácter regulado del mercado profesional, las licencias o autorizaciones de creación de instituciones siempre han tenido una alta dependencia del sistema político. Como en América Latina, se verifica una alta participación de políticos de centro y de derecha en la propiedad de las instituciones universitarias, en tanto la autorización de su funcionamiento era una potestad y un acto administrativo del poder político.

El cambio Constitucional y la derivación normativa en la ley de educación (LDB) de 1996, facilitó retomar e incrementar la tendencia histórica de expansión de la educación privada, al contribuir a un aumento de la diversificación y a un funcionamiento de la educación superior con fines de lucro, lo cual permitió niveles de flexibilidad y de gestión que facilitaron un nuevo ciclo expansivo. El sector privado tuvo en el largo plazo un crecimiento persistente desde el 43,7\% de cobertura en 1933, al 69\% en el 2001 y 74,6\% en el 2007. ${ }^{10}$ Salvo por una leve caída del sector privado entre 1980 y 1995 , en el marco de fuertes presiones y luchas políticas, siempre hubo un decrecimiento relativo del sector público en relación a la cobertura en el grado frente al sector privado por tener componentes estructurales asociado al modelo de investigación de la universidad pública. Sin embargo, desde la LDB hay un cambio de dimensión significativo. Para algunos especialistas la característica más distintiva de la educación superior en el periodo 19952007 es "la expansión libre y de dudosa calidad del sector privado con fines de lucro" (Díaz Sobrinho, Regina F de Brito, 2008). ${ }^{11}$ Ello se dio en el marco de contexto de una creciente y nueva diferenciación institucional de fines de los 80 y 90, gracias a la cual el 
sector privado creció asociado a ofertas destinadas a sectores de menos ingresos, de estudios no universitarios (modelo de colegios americanos), así como de expansión de instituciones regionales, que eran tanto las demandas de los mercados laborales como las posibilidades de pago de los sectores de menores ingresos. Además, la LDB permitió una mayor diferenciación institucional a partir del desarrollo de los Centros Universitarios. La Constitución había establecido que la Universidades estaban obligadas a realizar tareas indisociablemente de investigación, docencia y extensión. La LDB facilitó la diferenciación al habilitar estas nuevas formas organizaciones institucionales, que tenían la posibilidad de ofrecer acceso a la educación superior privada a menores costos, al no requerirse la obligatoriedad de realizar investigación y extensión junto a la docencia de grado. Al tiempo, solo las Universidades pueden crear cursos directamente sin requerir la autorización previa, imponiéndose nuevas modalidades de regulación sobre las otras instituciones. Así, la tipología institucional se estructuró a partir de la existencia de Universidades, Centros Universitarios, e Instituciones no Universitarias (Facultades, Facultades aisladas, Instituciones Superiores de Educación, Centros Federales de Educación Técnica (públicos solo) y Centros de Educación Tecnológicos. La LDB también habilito nueva diferenciación dada por la educación a distancia que ha permitido en el tempo un aumento de este sector. Iniciada a fines del siglo XX, alcanzó al $7 \%$ de la matrícula total en el 2007, al alcanzar a 369.766 estudiantes para ese año.

La nueva tipología establecida permitía la creación de instituciones de educación privadas que podían tener fines de lucro (pagando impuestos) e instituciones sin fines de lucro que podían ser comunitarias, filantrópicas o confesionales. El funcionamiento de la educación superior con fines de lucro permitió el ingreso de nuevos sectores oferentes, fuertemente motivados por la búsqueda de beneficios económicos, lo cual facilitó el fuerte ciclo expansivo de instituciones a través de la incorporación de nuevas modalidades de gestión y del ingreso de importantes capitales nacionales y extranjeros. La gestión empresarial promovió altos niveles de flexibilidad en la gestión y en las dinámicas pedagógicos, a partir de la conformación de grupos cada vez más amplios en términos de su cobertura regional. El nuevo marco legal promovió la transición del sector desde una dinámica dominante basada en el paradigma del bien público y sin fines de lucro, de instituciones familiares, de pequeñas instituciones individuales, hacia uno nuevo marcado por la mercantilización, la búsqueda del lucro y de grandes grupos educativos a nivel nacional. ${ }^{12}$

El cambio constitucional a través del Art. 209 dispuso que "la enseñanza es libre a la iniciativa privada, atendiendo a las siguientes condiciones: I. cumplimiento de las normas generales de la educación nacional; II autorización y evaluación de calidad por el Poder Público". ${ }^{13}$ Tal marco facilitó el establecimiento de la educación con fines de lucro así como también estableció un rol superior del Estado en la supervisión y control de la educación superior que se aprobaron en la Ley de educación de 1996. Así, asociado a este proceso, se comenzó a producir un aumento del nivel de regulación público sobre el sistema de educación superior, y fundamentalmente sobre las instituciones privadas de educación superior. El aumento de la diferenciación que produjo la dinámica del lucro, también promovió un incrementó del nivel regulador, el cual a su vez incrementa las imperfecciones del mercado, y torna más complejo el funcionamiento de los mercados universitarios que se pueden tornar más burocratizados y regulados. Así, por una parte se liberó el funcionamiento de los mercados sobre la base de lógicas mercantiles, tanto orientadas al lucro como sin fines de lucro, y por la otra se sentaron las bases de un aumento del nivel de regulación, que crecientemente se ha dado en forma altamente centralizada y politizada. Así, la diferenciación y la incorporación de la modalidad del 
lucro en la gestión universitaria, derivó en un aumento de la regulación, una de cuyas manifestaciones ha sido el establecimiento de mecanismos de aseguramiento de la calidad.

El cambio se dio a través de la nueva legislación aprobada en el año 1996 (Directiva y Bases de la Educación Nacional (LDB) aprobada en concordancia con la aprobación de una nueva Constitución en el año 1988 que dispuso que la "educación es libre a la iniciativa privada" sujeta a las leyes y a la autorización y la evaluación de la calidad, y de cuya concepción se aprobó la creación de sistemas de evaluación y de acreditación de la educación. Asociado a ese cambio, durante el periodo de Fernando Henrique Cardoso (1995-2002) se produjo el fuerte proceso de expansión de instituciones y de la matricula de la educación superior privada, lo cual impulsó a su vez un aumento de la matrícula de la educación superior en general. Sin duda, las fuertes demandas de acceso no se podían canalizar hacia un sector público, tradicionalmente altamente selectivo y donde además el financiamiento público era escaso para permitir esa absorción, lo cual reafirmó que el crecimiento de la cobertura se realizase por la vía dominantemente del sector privado.

\section{Dimensión académica como instrumento de regulación}

La LDB incrementó el proceso de diferenciación de la educación superior brasilera, no sólo al desarrollar una nueva tipología institucional y al promover la existencia de instituciones universitarias sin la obligatoriedad de tener que ofertar postgraduación de "stricto sensu" y además no tener que realizar investigación, sino además al promover la existencia de instituciones privadas con fines de lucro. El nuevo escenario al aumentar el nivel de diferenciación institucional contribuyó a facilitar a su vez el aumento de las demandas al tiempo que un aumento de la oferta, así como también facilitó una mayor relación de la oferta con los mercados laborales y desestructurando la tradicional separación entre mercado educativo y mercado laboral. El mayor impacto fue un avance hacia el desarrollo de currículos por competencia que promovió la propia LDB.

Esta al tiempo que facilitó la oferta educativa al establecer la libertad de enseñar y aprender y la coexistencia en la prestación del servicio entre instituciones públicas y privadas (Articulo 3), estableció una regulación permanente al disponer que "la autorización y el reconocimiento de cursos, así como el licenciamiento de instituciones de educación superior, tendrán plazos limitados, siendo renovados periódicamente después de un proceso regular de evaluación" (Art. 46)

El nuevo marco de diferenciación legal, facilitó entonces la canalización de la creciente presión de nuevos egresados de la educación media. Entre 1980 y 1995 la tasa de escolarización bruta de la educación media aumentó un 50\% al pasar de 33,3\% al 47\%, y continuó trepando para alcanzar al 74,8\% en 1999. La tasa de escolarización líquida por su parte pasó del $14,3 \%$ en 1980 al $32.6 \%$ en $1999 .{ }^{14}$

Uno de los ejes de la política del gobierno de Lula iniciado en el 2003 se ha focalizado en un aumento de la regulación sobre el sector privado a partir de un enfoque de calidad. Para algunos autores, al mismo tiempo que los gobiernos han fomentado una cierta liberalización de los sistemas educativos -aunque con formas diferentes en los distintos países esta tendencia se contrabalancea con otra de control, generalmente curricular o evaluativa. Durante el periodo de Fernando Enrique Cardozo ello se inició tímidamente, sin embargo a partir del 2003 se produce un cambio de entonación respecto a la libertad de funcionamiento del sector privado propendiendo tal vez a la búsqueda de un re-equilibrio de la relación entre la educación privada y la pública que desde la LDB de 1996 se alteró en $10 \%$ porcentuales, al pasar el sector privado de $60 \%$ a $70 \%$ entre 1996 y el 2003. Ello 
promovió una búsqueda de acotar la expansión de la educación privada a través de regulaciones de tipo académicas. Como se afirma "en su gobierno fue priorizada la evaluación de la educación superior, que era uno de los aspectos destacados en el Programa de Gobierno de la Coalición Lula presidente. La evaluación era considerada uno de los elementos relevantes tanto para garantizar la calidad del sistema como para promover la autonomía universitaria. (...) El Sistema Nacional de Evaluación de la Educación Superior (SINAES) fue la propuesta que finalmente con ajustes se aprobó como Ley 10,861 el 14 de abril de 2004 (...)La evaluación institucional está vinculada con la regulación porque sus resultados incidirían en los procesos de credenciamiento y recredenciamiento de las instituciones" Muy rápidamente en el 2004 se aprobó la ley de creación de la agencia de evaluación SINAES que implico un cambio en el modelo de evaluación de la educación superior en el Brasil, que anteriormente promovía el ranking y la competitividad. ${ }^{15}$

La regulación de SINAES ha sido el objeto de numerosas protestas por parte de la educación superior privada en Brasil que concibe que el instrumento no tenga como objetivo meramente la calidad sino el control sobre el sector privado. Los ejes de modelo de evaluación del SINAES son la articulación en el ámbito público centralizado de la autorización, la evaluación y la acreditación, en el marco de lo establecido en la Constitución que establece que la iniciativa privada esta sujeta a la autorización y evaluación de la calidad por el poder público. Se conforma como un modelo de agencia monopólica, estatal y centralizada, con un enfoque basado en criterios académicos exclusivamente y donde los pares y actores que evalúan al sector privado son exclusivamente del sector público. Para algunos académicos como Simon Schwartzman, "nenhuma agência nacional tem condições de desenvolver um sistema de avaliação que possa cobrir, de forma adequada e não burocrática, todas as 2270 instituições e mais de 22 mil cursos superiores presencias que existem no Brasil, sem falar nas novas modalidades de cursos a distância, semipresenciais, etc.". ${ }^{16}$

\section{Cuadro $\mathbf{N}^{\circ}$}

Matrícula de la educación superior en Brasil e incidencia del sector privado

\begin{tabular}{|l|c|c|c|c|c|c|c|c|}
\hline Año & $\begin{array}{c}\text { Matricula } \\
\text { total }\end{array}$ & Aumento & $\begin{array}{c}\text { Matricula } \\
\text { Sector } \\
\text { privado }\end{array}$ & \% del total & $\begin{array}{c}\text { Variación } \\
\text { absoluta }\end{array}$ & $\begin{array}{c}\text { Matricula } \\
\text { Sector } \\
\text { publico }\end{array}$ & \% del total & Aumento \\
\hline $\mathbf{1 9 9 1}$ & 1565056 & & 959320 & $61,29 \%$ & & 605736 & & \\
\hline $\mathbf{1 9 9 2}$ & 1535788 & $-0.01 \%$ & 906126 & $59,01 \%$ & $-5.54 \%$ & 629662 & $40.98 \%$ & $3,9 \%$ \\
\hline $\mathbf{1 9 9 3}$ & 1594668 & $3,8 \%$ & 941152 & $59,01 \%$ & $3.8 \%$ & 653516 & $40,98 \%$ & $3,7 \%$ \\
\hline $\mathbf{1 9 9 4}$ & 1661034 & $4,2 \%$ & 970584 & $58,43 \%$ & $3.1 \%$ & 690450 & $41,57 \%$ & $5,6 \%$ \\
\hline $\mathbf{1 9 9 5}$ & 1759703 & $5,9 \%$ & 1059163 & $60,53 \%$ & $9,12 \%$ & 700540 & $39.46 \%$ & $1.46 \%$ \\
\hline $\mathbf{1 9 9 6}$ & 1868529 & $6,2 \%$ & 1133102 & $60,64 \%$ & $6,9 \%$ & 735427 & $39,35 \%$ & $4.9 \%$ \\
\hline $\mathbf{1 9 9 7}$ & 1936625 & $3,6 \%$ & 1186433 & $61,26 \%$ & $4,7 \%$ & 750182 & $38,72 \%$ & $2.0 \%$ \\
\hline $\mathbf{1 9 9 8}$ & 2125958 & $9,7 \%$ & 1321229 & $62,14 \%$ & $11,36 \%$ & 804729 & $37.85 \%$ & $7,2 \%$ \\
\hline $\mathbf{1 9 9 9}$ & 2350945 & $10,58 \%$ & 1527923 & $64,99 \%$ & $15,64 \%$ & 823022 & $35.0 \%$ & $2.2 \%$ \\
\hline $\mathbf{2 0 0 0}$ & 2694245 & $14,6 \%$ & 1807219 & $67.07 \%$ & $18,27 \%$ & 887026 & $32.92 \%$ & $7.7 \%$ \\
\hline $\mathbf{2 0 0 1}$ & 3030754 & $12,48 \%$ & 2091529 & $69.01 \%$ & $15,73 \%$ & 939225 & $30.98 \%$ & $5.8 \%$ \\
\hline $\mathbf{2 0 0 2}$ & 3479913 & $14.82 \%$ & 2428258 & $69,77 \%$ & $16,09 \%$ & 1051655 & $30,2 \%$ & $11.97 \%$ \\
\hline $\mathbf{2 0 0 3}$ & 3887022 & $11,69 \%$ & 2750652 & $70,64 \%$ & $13,3 \%$ & 1136370 & $29.36 \%$ & $8.05 \%$ \\
\hline $\mathbf{2 0 0 4}$ & 4163711 & $7.11 \%$ & 2985405 & $71.70 \%$ & $8.5 \%$ & 1178328 & $28.29 \%$ & $3.69 \%$ \\
\hline $\mathbf{2 0 0 5}$ & 4453156 & $6,9 \%$ & 3260967 & $73.22 \%$ & $9,2 \%$ & 1192189 & $26.77 \%$ & $1.17 \%$ \\
\hline $\mathbf{2 0 0 6}$ & 4674694 & $4,9 \%$ & 3466407 & $74,15 \%$ & $6,3 \%$ & 1208287 & $25.87 \%$ & $1.35 \%$ \\
\hline $\mathbf{2 0 0 7}$ & 4880381 & $4,4 \%$ & 3639413 & $74,57 \%$ & $4.9 \%$ & 1256618 & $25,43 \%$ & $4.0 \%$ \\
\hline
\end{tabular}

Nota: El dato de 2007 es matrícula presencial y de graduación. Fuente: IEP. Seria histórica datos de SINAES. http://sinaes.inep.gov.br/sinaes/. Cálculos propios 


\section{Ejes políticos de la educación superior privada}

El desarrollo de la educación superior en Brasil ha estado marcado por un escenario de tensión y conflictos entre lo público y lo privado sobre la base de modelos diferenciados. Mientras que la lucha entre la universidad pública y privada en la región es desde los 80, en Brasil ya desde los años 20 el conflicto entre lo público y lo privado estaba posicionado en el campo educacional brasilero (Lerche; 1997), Tales tensiones se han expresado en los marcos de las políticas, tanto a nivel Constitucional y legal como en las múltiples normativas a nivel ejecutivo.

El equilibrio se dio en el reconocimiento de la libertad de enseñanza, pero sobre la base de la supeditación a la regulación pública, al incorporarse constitucionalmente la sujeción al control. Ello se asociaría a un modelo dual completo, al darse la gratuidad absoluta en el sector público y a la posibilidad de una educación con fines de lucro en el sector privado. La dimensión política de la educación superior, marcada por una génesis con un fuerte peso de la educación superior privada sin fines de lucro, sentó las bases de un sistema universitario fragmentado basado en la autonomía pública sobre base académica y gratuita, y de autoregulación privada autónoma sobre base mercantil de acceso, que duró durante varias décadas. Sin embargo, un cambio político significativo se dio con la incorporación de los fines de lucro a la educación, y al tiempo una menor libertad de gestión. Ello planteó la diferenciación respecto al desarrollo anterior de un modelo privado sin fines de lucro, menos diferenciado institucionalmente pero con más autonomía de gestión educativa.

Este modelo histórico de funcionamiento desde 1996 acompañado a la masificación de las demandas, ha comenzado a cambiar en los últimos años en el marco de la política del Gobierno de Lula que propende a alcanzar un 30\% de cobertura para la población de 18 a 24, a partir de la expansión del sector público sobre la base de las siguientes políticas:

1. Expansión de la educación a distancia del sector público a partir de la creación de una Universidad a distancia que articula, regula, promueve y financia la oferta de educación superior a distancia pública. La educación privada virtual ha tenido una fuerte expansión en las instituciones privadas como en la universidad de Paraná, UNISUL, PUC-PR y actualmente se tiende a acotar. ${ }^{17}$

2. Expansión de nuevas instituciones públicas con menos presupuestos por alumnos, localizadas en el interior de los Estados

3. Expansión de la cobertura de las instituciones públicas por la vía del aumento de la ofertas de cursos y plazas nocturnas, ajustado a la propia LDB de 1996 que establecía en el artículo 47 la obligatoriedad de la oferta de cursos nocturnos en las instituciones públicas, pero que recién se ha expandido en los últimos años, asociado a recursos financieros y a disposiciones específicas, tanto en las Universidades Federales como en las Universidades Estaduales

4. Aumento del acceso de sectores tradicionalmente excluidos en las instituciones públicas a partir del establecimiento en las instituciones públicas de políticas proactivas de cuotas fundamentalmente para estudiantes negros e indígenas.

5. Aumento de los controles de calidad sobre la educación privada. ${ }^{18}$ La regulación inclusive incorpora la clausura de instituciones.

6. Creación de un sistema de becas dotadas de un acceso especial para los sectores de menos ingresos, como contraparte de la exoneración del pago de los impuestos de las instituciones privadas. 
En ese contexto de cambio de la educación superior pública tiende a promover un cambio en sistema terciario, al propender a un aumento de la oferta pública y por ende una disminución de la presión de ingreso sobre la educación privada.

\section{Dimensión social de las universidades privadas}

En los últimos años y particularmente desde el 2003 con el nuevo gobierno, se ha iniciado una amplia discusión para aumentar la cobertura que en el caso de Brasil se ha focalizado en política de cotas de las Universidades, y por las cuales varias universidades estaduales y federales han establecido acceso preferenciales para población negra e indígena. Al tiempo, la Cámara de Diputados aprobó un proyecto a fines del 2008 luego de una larga discusión que se inició en el Congreso en 1999 y el cual aun está en trámites en el senado. El proyecto aprobado establece que "la mitad de las plazas de las universidades federales y de las escuelas técnicas federales sería reservada para alumnos oriundos de las escuela públicas. De ese conjunto a su vez la mitad se reservaría para estudiantes con renta familiar per. cápita de hasta la mitad del salario mínimo. Las cotas raciales incidirían sobre las dos mitades: parte de las plazas serían reservadas para alumnos negros o indígenas de acuerdo con la representación de cada Estado". ${ }^{19}$ El proyecto inicial no incluía cotas raciales sino que se focalizaba en cupos para el acceso de estudiantes procedentes de las escuelas públicas y en la dinámica política se ha ido focalizando en la población negra. Esta transformación de los accesos al sector público brasilero tenderá a producir cambios significativos en el sector privado. Tal nueva estrategia educativo implicará a su vez un cambio en múltiple dimensiones. Además del reposicionamiento del sector público y del privado, implicaría cambios sociológicos enormes. Para algunos, se estaría solucionando el problema de las acceso de sectores excluidos, pero creando un nuevo problema a partir de la "racialización" del Brasil y del derecho brasilero. ${ }^{20}$ En el año 2009 la Suprema Corte del Estado de Río de Janeiro declaró inconstitucional la política de cotas que había desarrollado la Universidad del Estado de Río de Janeiro (UERJ) que había establecido un porcentaje del ingreso a la institución de los diversos sectores en función del porcentaje de la población del Estado. Esta declaración ha paralizado por lo pronto la discusión de la Ley.

\section{Dimensión económica de la educación superior}

La expansión de la educación superior privada se hizo exclusivamente sobre las rentas de los estudiantes y de sus familias, al carecer de sistemas significativos de financiamiento. Ello ha marcado el propio perfil social de sus estudiantes. "Apenas el 6,9\% de los estudiantes brasileros de las universidades privadas cuentan con algún tipo de financiamiento para pagar sus mensualidades. De los 3,6 millones de alumnos, solamente cerca de 250 mil utilizan el financiamiento estudiantil (Fies) del gobierno federal, de préstamos de las instituciones financieras o de programas de las propias instituciones. Además los beneficiarios de tales financiamientos se están reduciendo. En el 2005, alcanzaban al $8,1 \%$ de los universitarios de las instituciones privadas, en tanto que en el 2006, fueron $7,4 \% .^{21}$

Según el Censo da Educação Superior del 2007 el sector privado es responsable del $89 \%$ de las IES existentes; del 72\% de los cursos de graduación ofertados y del $75 \%$ de los alumnos matriculados en este nivel de educación en el país. Las instituciones de ES privadas empleaban en el 2007 más de 218 mil profesores y 173 mil funcionarios del sector técnico-administrativo, que significa cerca de $65 \%$ del total de recursos humanos en 
la educación superior brasilera pública y privada. Sin embargo es de destacar la fuerte existencia de micro universidades ya que actualmente, el $82 \%$ de las IES tienen menos de 2.000 alumnos matriculados, fundamentalmente en los municipios pequeños.

Tal escenario tiende a incidir en el actual enlentecimiento de la expansión de la educación superior privada que ya ha alcanzado a los sectores con recursos. "Há uma enorme falta de linhas de financiamento adequadas para esses jovens", afirma Hermes Figueiredo, presidente del SEMESP (Sindicato das Entidades Mantenedoras de Estabelecimentos de Ensino Superior no Estado de São Paulo). Según el "a maior dificuldade dos alunos está na obtenção do crédito, que exige fiador e tem juros considerados altos pelos estudantes. A taxa de inadimplência do Fies, por exemplo, está em 20\%, índice semelhante à inadimplência nas mensalidades das faculdades particulares, que gira em torno de $23 \%$. Ainda assim, o Fies responde por $67,6 \%$ dos 250 mil alunos com crédito. Outros $20 \%$ contam com programas das próprias instituições onde estudam".

El mercado parece estar creciendo a tasas menores, resultado de la ausencia de mecanismos de financiamiento a las matrículas y un cierto agotamiento de los mercados de altos ingresos. Ello pudiera estar incidiendo en la reducción en la tasa de creación de nuevas instituciones de educación superior particulares. ${ }^{22}$ Este contexto de desaceleración se verifica en los números registrados por el Censo de Educación Superior 2007 del INEP, ya que de acuerdo con sus relevamientos, apenas once IES fueron creadas entre 2006 y 2007, diez de ellas privadas y apenas una pública, lo que representa una expansión de apenas 0,48\%, fuertemente diferenciado de la expansión del período de 1997 a 2007, cuando el crecimiento fue del $153,44 \%$.

Cuadro $\mathbf{N}^{\mathbf{0}}$

\begin{tabular}{|c|c|c|c|c|c|c|}
\hline \multicolumn{7}{|c|}{ Evolución del número de instituciones de ES } \\
\hline & General & Crecimiento & Públicas & Crecimiento & Privadas & Crecimiento \\
\hline $\mathbf{1 9 9 4}$ & 851 & & 218 & & 633 & \\
\hline $\mathbf{2 0 0 0}$ & 1180 & - & 176 & - & 1004 & - \\
\hline $\mathbf{2 0 0 1}$ & 1391 & $17,88 \%$ & 183 & $3,98 \%$ & 1208 & $20,32 \%$ \\
\hline $\mathbf{2 0 0 2}$ & 1637 & $17,69 \%$ & 195 & $6,56 \%$ & 1442 & $19,37 \%$ \\
\hline $\mathbf{2 0 0 3}$ & 1859 & $13,56 \%$ & 207 & $6,15 \%$ & 1652 & $14,56 \%$ \\
\hline $\mathbf{2 0 0 4}$ & 2013 & $8,28 \%$ & 224 & $8,21 \%$ & 1789 & $8,29 \%$ \\
\hline $\mathbf{2 0 0 5}$ & 2165 & $7,55 \%$ & 231 & $3,13 \%$ & 1934 & $8,11 \%$ \\
\hline $\mathbf{2 0 0 6}$ & 2270 & $4,85 \%$ & 248 & $7,36 \%$ & 2022 & $4,55 \%$ \\
\hline $\mathbf{2 0 0 7}$ & 2281 & $0,48 \%$ & 249 & $0,40 \%$ & 2032 & $0,49 \%$ \\
\hline Total & & $\mathbf{9 3 , 3 1 \%}$ & & $\mathbf{4 1 , 4 8 \%}$ & & $\mathbf{1 0 2 , 3 9 \%}$ \\
\hline
\end{tabular}

A pesar del cambio en las reglas de apertura de las instituciones que fueron modificadas al termino del período de Cardoso (1995-2002) y al inicio del gobierno de Lula, la reducción del ritmo de crecimiento especialmente del sector privado no sólo era prevista sino que marca un nuevo contexto. La reducción del crecimiento ha tenido varios enfoques. ${ }^{23}$ A opinión del secretario ejecutivo del Forum das Entidades Representativas do Ensino Superior Particular, Gabriel Mario Rodrigues es diferente: para él la desaceleración no indica crisis sino una consolidación del sector privado en el sistema educativo, en tanto adecuación de la oferta a la demanda, en tanto que la reducción del crecimiento es natural en cualquier sector económico en la medida que el desarrollo se enmarca en las necesidades y demandas del mercado. Rodrigues sostiene que la cantidad de estudiantes oriundos de las clases A y B disminuyó lo que refleja a su vez la reducción de la demanda del sector privado. El mercado existe, pero carece de condiciones económicas, ya que está concentrado en las clases de menor poder adquisitivo. La saturación de la demanda de los 
sectores de altos ingresos y la existencia de una demanda insatisfecha en sectores de menores ingresos y de menores stocks de capital cultural, no sólo incide sobre la tendencia a la baja de los precios de las matrículas, sino también que presiona hacia la baja los niveles de calidad, y complejiza las escenarios de la gestión y de la calidad.

Uno de los claros problemas que atraviesa la dinámica de la alta cobertura privada en Brasil está asociado a los problemas que implica el ingreso de sectores sociales de menores ingresos. Según un informe de SEMESP, la tasa de deserción de las facultades privadas de la región metropolitana de San Pablo creció $40 \%$ y alcanzó a 20,21\% en el año 2007, o sea un quinto de los alumnos matriculados en ese año. Según el análisis de Semesp, el aumento de la inclusión de alumnos de las clases C e D, con más dificultades financieras para mantener los cursos es lo que explica el aumento de la deserción.24 Tal contexto aumenta la presión sobre la disminución de la tasa de incremento, y aumenta la presión para procesos de reformas en el sector privado y un mayor nivel de concentración como expresión de la búsqueda de mayor eficiencia para poder ofertar a menores costos. Para algunos especialistas muchas facultades no conseguirán sobrevivir a las plazas ociosas, al aumento de la competencia, a los altos costos de administración y a las crecientes disposiciones públicas de regulación de calidad, previéndose una retracción del número de IES. En opinión de Rodrigues, la consolidación del sector va a determinar que sólo las instituciones que mantienen buena infraestructura, cuerpo docente calificado, tradición de mercado y equilibrio financiero van a sobrevivir en este proceso. Ello esta derivando, en el marco de una economía mercantil y de modelos basados en fines de lucro, en un fuerte proceso de fusiones en tanto se constituye como alternativas de crecimiento en la competencia. Las fusiones no se expresan en los datos, ya que ellas no eliminan la contabilidad de las instituciones en forma separada. La concentración tiene un fuerte impulso focalizado en la gestión de las universidades con fines de lucro promovidas desde 1996.

Para el año 2008 las IES con fines lucrativos presentaban una participación significativa según el documento base de Forum Nacional de Ensino Superior. A partir de sus datos, para 1999 el sector privado con fines de lucro representaba el 27,48\% de la matrícula total y el $42.35 \%$ de la matrícula privada, en tanto que para el año 2006, el sector con fines de lucro era ya el $41.14 \%$ de la matrícula total y representaba el $55.49 \%$ del total de la matrícula privada. La concentración y las fusiones se conforman como los impulsores de la nueva fase del sector privado marcada por la expansión pública, las regulaciones de calidad, el agotamiento de los mercados de altos ingresos y la creciente lógica mercantil de centralización. La concentración es una derivación del mercado, que es a su vez incentivada por las propias políticas de control de calidad que van promoviendo una reducción del ingreso de nuevos proveedores y el aumento de los costos administrativos de gestión, facilitando a su vez gestiones más profesionales y por ende menos académicas o familiares. La respuesta a la expansión pública y como derivación del propio desarrollo de la ESP es también la constitución de grupos empresariales universitarios que se focalizan en el segmento medio, y que se basan en nuevos modelos de gestión con centralización de la inscripción, del pago, de los aspectos académicos, de la evaluación, de los procesos administrativos de egreso, al tiempo que solo el proceso educativo se descentraliza y se focaliza en las sedes institucionales. La concentración institucional se conforma como una dinámica del proceso educativo, que al tiempo que se articula en micro y en grandes universidades. 


\section{Cuadro $\mathbf{N}^{\circ}$}

Concentración universitaria en Brasil

Las diez universidades con mayor matrícula del país

\begin{tabular}{|l|c|c|c|c|c|}
\hline \multicolumn{1}{|c|}{ Nombre } & Estado & Régimen & Sector & Matricula & Egreso \\
\hline Universidade Paulista & SP & Privada & & 145.498 & 14.789 \\
\hline Universidade Estácio de Sá & RJ & Privada & & 116.959 & 17.093 \\
\hline Universidade Nove de Julho & SP & Privada & & 84.398 & 4.573 \\
\hline Universidade Bandeirante de São Paulo & SP & Privada & & 69.074 & 9.385 \\
\hline Universidade Presidente Antônio Carlos & MG & Privada & & 57.291 & 10.953 \\
\hline Universidade de São Paulo (UNESP) & SP & Publica & Estadual & 49.774 & 6.734 \\
\hline Universidade Salgado de Oliveira & RJ & Privada & & 47.853 & 9.469 \\
\hline Universidade Luterana do Brasil & RS & Privada & Confesional & 43.620 & 5.670 \\
\hline $\begin{array}{l}\text { Universidade Estadual Paulista Júlio de } \\
\text { Mesquita }\end{array}$ & SP & Publica & Estadual & 32.204 & 5.803 \\
\hline Universidade Federal do Pará (UFPA) & PA & Publica & Federal & 32.094 & 4.557 \\
\hline Total de cobertura y egreso & & & & 678.765 & 89026 \\
\hline \% sobre el total nacional & & & & $\mathbf{1 3 , 9 \%}$ & \\
\hline
\end{tabular}

Fuente: Censo da Educação Superior 2007, MEC (Ministério da Educação). ANUP.

http://www.anup.com.br/noticiaDetalhe.php?id=5122P

La concentración de la educación superior brasilera es elevada y creciente desde 1996, y en el marco del agotamiento del modelo de crecimiento y del nuevo contexto político se tenderá a incrementar. Los datos no permiten visualizar claramente el fenómeno por la alta cantidad de micro universidades y la existencia de redes de propiedad común. Ya que el 82\% de las instituciones tienen menos de 2000 alumnos. Para 1994 la media era de 1533 alumnos por institución en el sector privado, para el 2000 alcanzó a 1800; en el 2004, 1669 y en el 2007, 1791. Ello muestra la gran diferenciación del sector privado. El sector público es en tanto más homogéneo, y la media de alumnos por institución que era de 3167 alumnos en 1994, alcanzó a 5040 en el 2000 y a 5047 en el 2007.

La concentración en pocos grupos es una tendencia histórica desde 1996 y que ha ganado fuerza desde 2007, cuando grupos privados importantes, como Anhanguera, con 110 mil alumnos, y Estácio de Sá, con 190 mil, fueron a la bolsa de valores, e emitieron acciones con el objeto de captar recursos para la adquisición de instituciones. Un estudio realizado por LatinLink, concluyó que en el sector educacional brasilero el grado de concentración será tan grande que para el 2015, los 20 mayores grupos estarán respondiendo por el $70 \%$ de los alumnos de la educación superior privada y que el $30 \%$ restante estarán distribuidos en facultades de menor tamaño con actuación solo local o regional. ${ }^{25}$ El estudio registra que las Facultades u otras modalidades institucionales menores que tengan que competir con los grandes grupos serán absorbidas, o desaparecerán por incapacidad de operar en los contextos de restricción de la demanda. Es parte de un proceso de racionalización derivado de la proliferación de instituciones y del aumento de los niveles de competencia.

Fabio Reis con las informaciones de The Chronicle (Almanac Issue 2010-2011), y del estudio referido de Hopper Educacional coincide en el nivel de concentración con los siguientes datos. ${ }^{26}$ 


\begin{tabular}{|l|c|}
\hline \multicolumn{2}{|c|}{ Ranking de Matrículas na Educação Superior Brasileira } \\
\hline Companhias de Educação Superior do Brasil & Estimativa de Matrículas 2008 \\
\hline Estácio Participaç̃es & 207.000 \\
\hline Di Gênio (UNIP + holding de 41 faculdades) & 197.000 \\
\hline Anhanguera Educacional & 130.000 \\
\hline Universidade Nove de Julho - Uninove & 92.000 \\
\hline Laureate International & 73.000 \\
\hline Grupo UNIBAN (Universidade Bandeirantes) & 70.000 \\
\hline Grupo UNIVERSO (Universidade Salgado de Oliveira) & 53.000 \\
\hline IUNI EDUCACIONAL & 46.000 \\
\hline Kroton & 43.000 \\
\hline Grupo Anima & 39.000 \\
\hline 10 GRUPOS EMPRESARIALES & 950.000 \\
\hline
\end{tabular}

Esta información permitiría sostener que apenas 10 grupos empresariales concentran el $26 \%$ de la matrícula privada. Incorporando los datos de educación a distancia donde la matrícula total alcanza a 5.8 millones de estudiantes, la concentración de estos grupos seria del entorno del $16 \%$ de la matrícula total.

Las fusiones y adquisiciones van a ocurrir en los segmentos más populares con mensualidades de hasta 400 reales, pues la escala pasó a ser el eje articulador de la calidad y de la producción sustentable en función de las crecientes políticas de control. El nivel de concentración se está aumentando por las fusiones y no por los crecimientos diferenciados como en el ciclo anterior. En 2008 hubo 55 adquisiciones, entre los cuales destaca la adquisición del grupo paranaense Campos Andrade que asumió el control de la Universidade Ibirapuera (Unib), de San Pablo. Este proceso también se asocia a fondos de inversión que ya tienen participación en grupos educativos importantes. ${ }^{27}$

Otra de las particularidades del modelo de educación superior del Brasil, por su tamaño, y su marco legal, ha sido el surgimiento de las universidades corporativa, que inclusive han creado la Associacao Brasileira de Educacao Corporativa. ${ }^{28}$ La universidad corporativa es una expresión de la expansión de la educación superior privada así como de la propia lógica de la competencia en el mundo de los negocios que impulsa al interior de las empresas a una búsqueda de desarrollar mayores ventajas competitivas por la vía de un aumento de la formación de sus trabajadores en el marco de una pertinencia más ajustada a sus características de demandas de competencias de esas empresas. La Universidad corporativa propende a una integración de la empresa con el trabajo y sus competencias de una forma más imbricada y donde la propiedad de la institución educativa pasa a ser de la empresa productora y que demanda personal capacitado.

Finalmente es de destacar en los últimos años en el marco del proceso de concentración, el ingreso de inversiones y grupos internacionales como el Grupo Laureate que a la fecha ha adquirido 9 instituciones de educación superior. ${ }^{29}$ El proceso de adquisición continua, cada vez más apoyado en recursos financieros de mercado captados en la bolsa o en créditos bancarios, apoyados en las inversiones preexistentes. Esta internacionalización favorecida por el nivel del mercado y el carácter de instituciones con fines de lucro dado a partir de la LDB de 1996, contribuye al propio proceso que incorpora la mercantilización, la concentración y la internacionalización que se constituyen en las dinámicas más significativas y que impulsan a su vez mejores niveles de calidad gracias a 
las escalas y a una gerencia más profesional al superarse la gestión de grupos familiares o empresarios independientes.

Notas:

${ }^{1}$ El presente artículo es parte de una investigación titulada "La nueva fase de la educación privada universitaria en América Latina (diferenciación, concentración, calidad, mercantilización e internacionalización)" que se desarrolló como línea de investigación en el marco de la Unidad de Investigación de la Facultad de Ciencias de la Educación de la Universidad de la Empresa entre septiembre 2009 y enero del 2011 y que se articuló en el marco del Programa de Pós-Graduação em Educação de la Faculdade de Educação de la Universidade Estadual de Campinas (Unicamp) (SP, Brasil) y contó con el Prof. Dr. Dermeval Saviani como interlocutor. La investigación en mayo del 2010 fue aprobada por la Comissão de Pós-Graduação que emitió el certificado de trabajo posdoctoral.

${ }^{2}$ Economista (UCV); Especialista en Marketing (UCUDAL); Especialista en Telemática e Informática para la Educación a Distancia (UNA); Magíster en Gerencia de la Educación (UJMV); Doctor en Ciencias de la Educación (UNESR); Doctor en Derecho (UBA); Certificado de Estudios Postdoctorales (UNESR); Certificado de Estágio de Pós-doutorado (UNICAMP). Fue director del Instituto Internacional de la UNESCO para la Educación Superior en América Latina y el Caribe (IESALC). Ha recibido en dos ocasiones el Premio Nacional de Literatura del Uruguay por sus trabajos. Actualmente es Decano de la Facultad de Ciencias Empresariales e investigador en el Doctorado en Educación de la Facultad de Ciencias de la Educación de la Universidad de la Empresa (Uruguay); e investigador en el Sistema Nacional de Investigadores (SNI) de la Agencia Nacional de Investigación e Innovación (ANII) del Uruguay.

${ }^{3}$ Marback Neto, Guillherme (2007), “Avaliacao: instrumento de gestão universitaria”. São Pablo: Sumus Editora

${ }^{4}$ Lei n. 5.540, de 28 de novembro de 1968, regulamentada pelo Decreto 464, de 11 de fevereiro de 1969.

${ }^{5}$ Durham, Eunice (2003), "Educação superior, pública e privada (1808-2000), pp. 203 Brock, Colin y Schwartzman, Simon (2005), "Os desafios da educação no Brasil”, Editora Nova Fronteira: SP

${ }^{6}$ Lerche, Sofia, "O público e o privado nas tramas de LDB", em Brzezinski, Iria (org.) (1997), "LDB interpretada: diversos olhares se entrecruzan", Cortez Editora: SP

${ }^{7}$ A Campanha Nacional de Aperfeiçoamento de Pessoal de Nível Superior (atual CAPES) foi criada em 11 de julho de 1951, pelo Decreto ${ }^{\circ} 29.741$, com o objetivo de "assegurar a existência de pessoal especializado em quantidade e qualidade suficientes para atender às necessidades dos empreendimentos públicos e privados que visam ao desenvolvimento do país". A CAPES é reconhecida como órgão responsável pela elaboração do Plano Nacional de Pós-Graduação Stricto Sensu, em 1981, pelo Decreto nº 86.791. É também reconhecida como Agência Executiva do Ministério da Educação e Cultura junto ao sistema nacional de Ciência e Tecnologia, cabendo-lhe elaborar, avaliar, acompanhar e coordenar as atividades relativas ao ensino superior. A Lei $\mathrm{n}^{\circ}$ 8.405, de 09 de janeiro de 1992, autoriza o poder público a instituir a CAPES como Fundação Pública Desde 1995, a CAPES passa por uma reestruturação, fortalecida como instituição responsável pelo acompanhamento e avaliação dos cursos de pós-graduação strictu sensu brasileiros. http://www.capes.gov.br/sobre-a-capes/historia-e-missao

8 "Um aluno do curso superior matriculado em uma universidade pública federal custa aos cofres públicos R\$ 27.420 anualmente, o que representa cinco vezes mais o custo de um aluno matriculado no setor privado". "Análise Setorial do Ensino Superior Privado do Brasil" de 2009 produzido pela Hoper Educacional, citado em Brasil e Estados Unidos: um olhar sobre os sistemas de educação setembro 18th, 2010 http://www.fabiogarciareis.com/wp/

${ }^{9}$ Entre estas instituciones se puede referir a FUVEST (Fundação Universitário para o Vestibular) responsável pelas provas da USP, Faculdade de Medicina da Santa Casa e Academia de Polícia; VUNESP (Fundação para o Vestibular da Universidade Estadual Paulista) que, além da UNESP, realiza vestibulares também para as Universidades Federais de São Carlos, de São Paulo, do Triângulo Mineiro, do ABC e para a Universidade Estadual de Ciências da Saúde de Alagoas (UNCISAL); a Fundação CESGRANRIO, que também faz vestibulares para várias instituições. Já a UNICAMP, por exemplo, organiza os próprios vestibulares por meio da COMVEST (Comissão Permanente para os Vestibulares).

${ }^{10}$ Censo e Sinopsis Estadisticas do Ensino Superior, MEC. 
11 "La educación Superior en Brasil: Principales Tendencias y Desafíos, Revista Avaliacao, (Campinas), V. $3 \mathrm{~N}^{\circ}$ 2, julio 2008. http://www.scielo.br/pdf/aval/v13n2/11.pdf (Revisión:_17/1/ 2009)

${ }^{12}$ Magalhaes Castro, Maria Helena de, "Estado e mercado na regulação da educação superior", em Brock, Colin y Schwartzman, Simon (2005), “Os desafios da educação no Brasil”, pp. 241, Editora Nova Fronteira: SP.

${ }^{13}$ http://www.acnur.org/biblioteca/pdf/0507.pdf (revisión 29 de setiembre 2009.

14 Durham, Eunice (2003), "Educacao superior, pública e privada (1808-2000), en Brock, Colin y Schwartzman, Simon (2005), “Os desafios da educacao no Brasil”, pp. 219. Editora Nova Fronteira: SP

${ }^{15}$ Barreyro, Gradys Beatriz (2006). "Evaluación de la educación superior brasileña: el Sinaes". Revista de la Educación Superior, enero-marzo, año/vol. XXXV (1), № 137, Asociación Nacional de Universidades e Instituciones de Educación Superior (ANUIES). DF, México pp. 63-73. http://redalyc.uaemex.mx/redalyc/html/604/60413705/60413705.html Revisión 16 de setiembre del 2009

16 Schwartzman, Simon, "O "conceito premiliminar" a as boas praticas de avaliação do ensino superior". Revista Estudios, Año 26, N 38, Dez de 2008

${ }^{17}$ Ver Patricia Torres Lupion y Joao Vianney en el estudio sobre Brasil publicado en Facundo, Angel; Mena, Marta; Rama, Claudio (2008). "Normativa de la educación a distancia en América Latina". Bogotá: UNAD, Virtual Educa, ICDE

${ }^{18}$ Para analizar el cambio en las modalidades de los procesos de evaluación y acreditación desarrollado con la Ley de SINAES del 2004, ver por ejemplo el trabajo de Beatriz Barreiro http://redalyc.uaemex.mx/redalyc/pdf/604/60413705.pdf) (Consulta realizada el 17 de enero del 2009), en el cual plantea un cambio en el paradigma de evaluación desarrollado desde el 1995 que considera de corte neoliberal. Revista de Educación Superior, ANUIES, México, № 137, enero-marzo 2006, pp.63-73

${ }^{19}$ O Estado de Sao Pablo, 19 de marzo del 2009, Pág. A6 "Grupo de senadores quer tirar critério racial de cotas"-

20 idem

${ }^{21}$ Datos de Semesp com base nos dados do Censo da Educação Superior de 2007, do Ministério da Educação (MEC). Portal Globo - 03/06/2009. "Só 7\% dos alunos de particulares têm bolsa, diz estudo". http://www.anup.com.br/noticiaDetalhe.php?id=5766

22 “Os dados que apontam essa tendência estão no Censo da Educação Superior 2007, divulgado pelo Inep (Instituto Nacional de Estudos e Pesquisas Anísio Teixeira). Segundo o levantamento, entre 2004 e 2007, 268 instituições privadas de Ensino Superior foram criadas, o que indica que o crescimento do setor no período foi de aproximadamente 13\%. Entretanto, quando comparado aos dados do quadriênio anterior, a desaceleração fica evidente, já que entre 2000 e 2003, 833 instituições surgiram, índice de crescimento de $70 \%$ ". ANUP News Letter n $42-13 / 2 / 2009$

${ }^{23}$ Hoper Educacional visualizaba que en 1996 el aumento de las instituciones reflejaba una demanda reprimida, y ello atrajo inversiones fuera del sector que provocó una expansión acelerada superior a la necesaria. ANUP. Por su parte el Sindicato das Entidades Mantenedoras de Estabelecimentos de Ensino Superior no Estado de São Paulo, considera que los datos indican que el sector privado estaría en crisis por la apertura excesiva y sin planificar de las instituciones privadas. Tal posición se basa en el número de "vagas" ociosas en el sistema particular. Los datos revelan que de las 2.216.977 vagas ofrecidas por las instituciones privadas en el año 2007, 51,2\% no fueron ocupadas, mostrando que la demanda es muy inferior a la oferta y que el mercado esta saturado.

${ }^{24}$ Folha de Sao Pablo. “Evasão nas faculdades particulares atinge índice recorde” - 01/06/2009

25 Bolha Global: Crise incentiva aquisição no ensino superior. Correio Brasiliense - 17/03/2009 | 18 mar 2009

26 Brasil e Estados Unidos: um olhar sobre os sistemas de educação. setembro 18th, 2010. http://www.fabiogarciareis.com/wp/

27 “GP Investimentos, comprou 20\% da Estácio de Sá; o Fundo Pátria, que participa do capital da Anhanguera, e UBC pactual, com 38\% da Faculdade do Nordeste (Fanor), em Fortaleza. Entre os fundos 
estrangeiros que já fincaram os pés no Brasil estão o americano Capital Group, acionista do grupo Kroton educacional, e o Cartesian Group, que adquiriu parte do grupo nordestino Maurício de Nassau. Já a Laureate Education, uma das maiores instituições privadas de ensino do mundo, tascou o controle da Faculdade Anhembi Morumbi”. Bolha Global: Crise incentiva aquisição no ensino superior". Correio Brasiliense 17/03/2009 | 18 mar 2009

28 Dekker de Caon, Korine, y de Souza Pinto, Marli Dias. "Cenarios e tendencias da universidades corporativa brasileira" en Colossi, Nelson; Souza Pinto, Marli (Org) (2004). Estudos e perspectivas em gestao universitaria", INPEAU: Brasil,

29 Business School São Paulo (BSP), Centro Universitário do Norte (UniNorte), Escola Superior de Administracão Dereito e Economia (ESADE), Faculdade dos Guararapes (FG), Faculdade Potiguar da Paraíba (FPB), Faculdade Unida da Paraíba (UniPB), Uni IBMR, Universidade Anhembi Morumbi (UAM), Universidade Potiguar (UnP). http://www.laureate-inc.com/univCampusBased.php (Revisión: 7/4/2010)

Recebido em: $\quad 21 / 03 / 11$

Aprovado em: $\quad 22 / 04 / 11$ 